\title{
COMMUNICATION
}

\section{Stomatite géographique : à propos de deux localisations palatines}

\section{Campana $\mathrm{F}^{1}$, Albertini AF${ }^{2}$, Fricain $\mathrm{JC}^{3}$}

1 - Centre Massilien de la Face. Marseille

2 - Cabinet d'anatomocytopathologie Medipath, Mougins

La langue géographique est une affection bénigne se manifestant par des macules érythémateuses de la langue. La stomatite géographique correspond à une localisation extra-linguale. Elle est rare et concernent généralement les joues et le plancher buccal. Nous rapportons ici deux cas de localisations palatines, dont une isolée.

\section{Observation}

Cas 1 : une femme de 53 ans consultait pour l'apparition d'une lésion palatine d'apparition récente. L'examen retrouvait une macule érythémateuse entourée d'un bord circiné blanc au niveau du palais dur droit. II existait des macules érythémateuses sur la langue évocatrice d'une langue géographique. L'interrogatoire retrouvait une notion de stress personnel récent. Le diagnostic de langue et palais géographique était posé. La patiente était revue à 15 jours et les lésions avaient disparu.

Cas 2 : un homme de 47 ans se présentait pour des lésions érythémateuses du palais dur et mou dans un contexte d'antécédent de purpura rhumatoide (PR) et de suspicion de maladie de Crohn (MC). L'examen retrouvait des lésions palatines éryhtémateuses entourées d'un liseré circiné blanchâtre évoquant un palais géographique. L'histologie excluait une MC ou un PR et était compatible avec ce diagnostic.

\section{Discussion}

La glossite exfoliatrice marginée ou langue géographique (LG) se définit cliniquement par une lésion eryhtémateuse entourée d'un bord blanchâtre surélevé et ayant tendance à migrer. La classification de Hume définit les différentes formes cliniques : le type 1 est la forme classique avec une atteinte uniquement linguale et des lésions caractéristiques migratrices. Le type 2 correspond de l'association des lésions de type 1 à des lésions extra linguales. Le type 3 s'observe avec des lésions linguales non caractéristiques qui peuvent ou non s'accompagner d'autres atteintes buccales. On distingue une forme fixe (lésions non migrantes et récidivantes au même endroit) et une forme abortive (lésion débutant en plaque blanchâtre et disparaissant avant d'atteindre l'aspect typique de LG). Le type 4, rare, correspond à une atteinte buccale ectopique isolée. On parle alors de stomatite géographique (SG). Selon cette classification, le cas 1 était un stade 2 et le cas 2 un stade 4 . Les localisations ectopiques sont classiquement les joues et le plancher buccal, la localisation palatine et en muqueuse kératinisée est exceptionnelle. Histologiquement, on retrouve une kératose de surface associée à un allongement des crêtes épithéliales, un infiltrat inflammatoire du conjonctif superficiel et la migration de polynucléaires 
neutrophiles dans l'épithélium formant des micro-abces. Ces lésions sont proches de celles retrouvées dans le psoriasis. Toutefois l'association LG-SG et psoriasis est encore mal comprise. Même si des brûlures sont souvent associées, les lésions géographiques ne nécessitent généralement pas de traitement, si ce n'est rassurer le patient.

\section{Références}

J Katz, A Shenkman, F Stavropoulos, E Melzer. Oral signs and symptoms in relation to disease activity and site of involvement in patients with inflammatory bowel disease. Oral Diseases 2003 ; 9 : 34-40.

U Mattsson et al. Oral psoriasis- a diagnostic dilemma : a report of two cases and a review of the literature. Oral Surg Oral Med Oral Pathol Oral Radiol 2015; 120: e183-e189.

Younai FS et al. Oral mucositis with features of psoriasis : report of a case and review of the literature. Oral Surg Oral Med Oral Pathol Oral Radiol Endod. 1997; 84:61-7. 